EPJ Web of Conferences 16, 03004 (2011)

DOI: $10.1051 /$ epjconf/20111603004

(C) Owned by the authors, published by EDP Sciences, 2011

\title{
Phase mask coronagraphy at JPL and Palomar
}

\author{
E. Serabyn ${ }^{\mathrm{a}}$ and D. Mawet \\ Jet Propulsion Laboratory, California Institute of Technology, 4800 Oak Grove Drive, \\ Pasadena, CA 91109, USA
}

\begin{abstract}
For the imaging of faint companions, phase mask coronagraphy has the dual advantages of a small inner working angle and high throughput. This paper summarizes our recent work in developing phase masks and in demonstrating their capabilities at JPL. Four-quadrant phase masks have been manufactured at JPL by means of both evaporation and etching, and we have been developing liquid crystal vortex phase masks in partnership with a commercial vendor. Both types of mask have been used with our extreme adaptive optics well-corrected subaperture at Palomar to detect known brown dwarf companions as close as $\sim 2.5 \lambda / \mathrm{D}$ to stars. Moreover, our recent vortex masks perform very well in laboratory tests, with a demonstrated infrared contrast of about $10^{-6}$ at $3 \lambda / \mathrm{D}$, and contrasts of a few $10^{-7}$ with an initial optical wavelength device. The demonstrated performance already meets the needs of ground-based extreme adaptive optics coronagraphy, and further planned improvements are aimed at reaching the $10^{-10}$ contrast needed for terrestrial exoplanet detection with a space-based coronagraph.
\end{abstract}

\section{INTRODUCTION}

The detection of very faint planets near much brighter stars calls for the suppression of starlight. Starlight suppression with phase mask coronagraphy has the advantage of employing transparent focal plane masks, which results in small inner working angles, high throughput and a clear off-axis field of view. Because of these advantages, we have been pursuing the development of phase mask coronagraphy at JPL over the past several years. We initially developed four-quadrant phase masks [1-3], which were manufactured using both differential evaporation and etching techniques at JPL. We then moved on to optical vortex phase masks of the vector vortex type [4-6], which apply a spiral focal plane phase shift by means of a rotationally symmetric half-wave plate. The half-wave plate devices are manufactured out of cured liquid crystal polymers by JDS Uniphase [4-6]. In the following, we discuss our recent work with phase masks at JPL, including both laboratory results and initial on-sky testing using our extreme adaptive optics well-corrected subaperture at Palomar.

\section{LABORATORY DEVELOPMENTS}

Figure 1 shows the operation of a phase mask coronagraph, together with the phase structure inherent in four-quadrant phase masks [7] and optical vortex masks. In the former, alternating phases of 0 and $\pi$ radians are applied in the four quadrants of the focal-plane point spread function, while in the latter case, an azimuthal phase spiral reaching an even multiple of $2 \pi$ radians in a circuit about the center is used. In the vector vortex case [4-6], the phase spiral is generated by means of a rotationally symmetric half wave plate, which imposes azimuthal phase spirals onto the two circular polarization states (Fig. 2). In either case, the starlight in a subsequent pupil plane lies entirely outside the image of the original aperture, allowing it to be blocked by a slightly undersized pupil (Lyot) stop.

ae-mail: gene.serabyn@jpl.nasa.gov

This is an Open Access article distributed under the terms of the Creative Commons Attribution-Noncommercial License 3.0, which permits unrestricted use, distribution, and reproduction in any noncommercial medium, provided the original work is properly cited. 


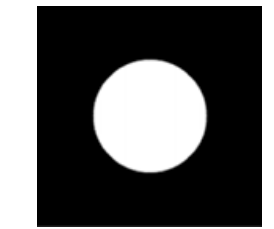

Input
pupil

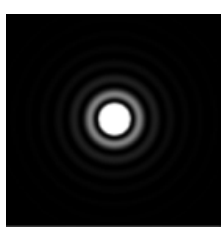

Focal plane

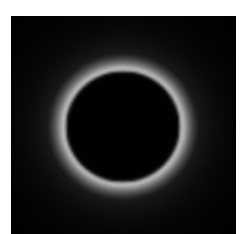

Pupil plane post-

vortex

pupil

\section{Image}

plane

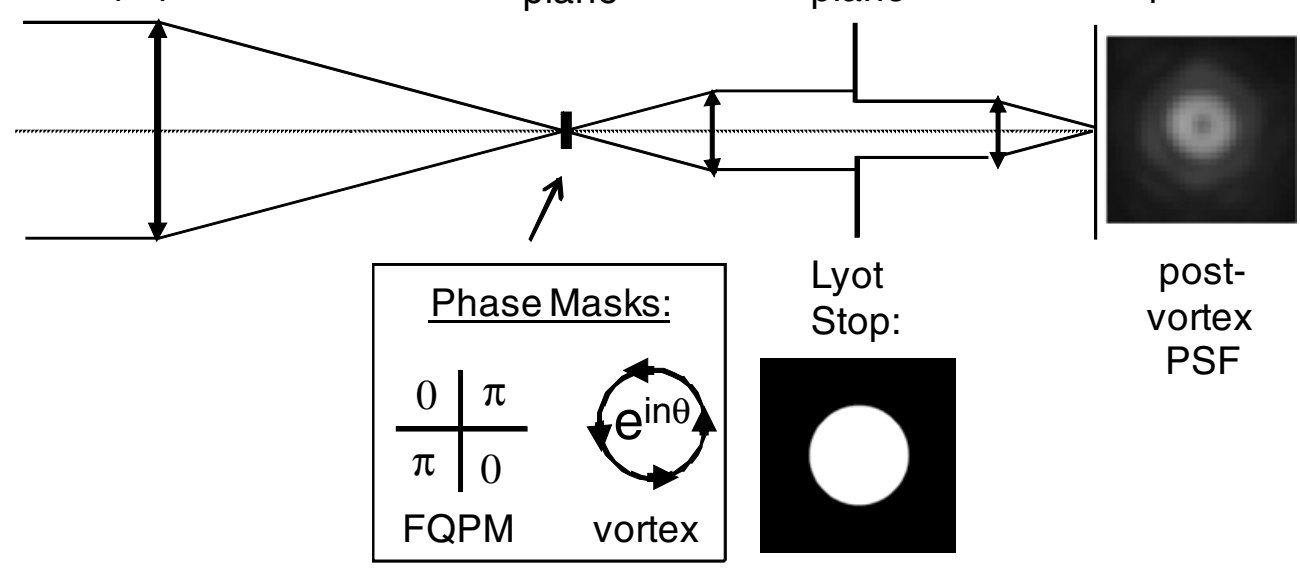

Figure 1. Coronagraph operation. After passage through the center of a focal plane phase mask, the starlight in a subsequent pupil plane lies outside the image of the aperture, where an undersized stop can remove it. The Lyot and focal plane images shown give the distributions for a vortex phase mask.

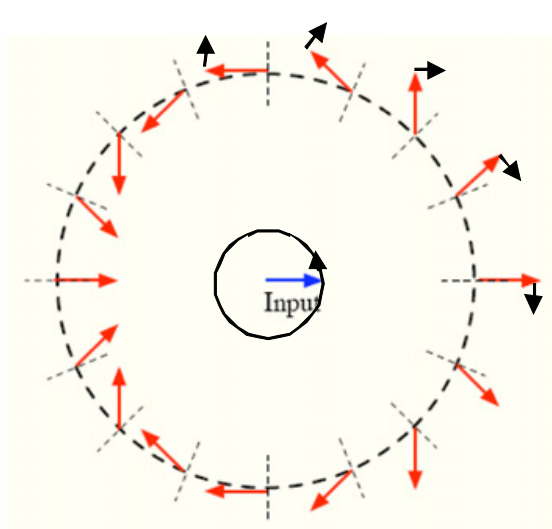

Figure 2. The generation of a spiral phase ramp by a vector vortex mask [4-6]. The local halfwave plate axes are shown by the short dashed lines, and the output fields for an $\mathrm{x}$-directed input field are shown by the long arrows around the periphery. The direction of rotation for one circular polarization state is shown by the short arrows (the other circular polarization state rotates in the opposite sense). The different instantaneous rotation angles correspond to the vortex's azimuthal phase spiral.

Figure 3 shows the off-axis contrast measured with one of our recent infrared vector vortex devices in the laboratory [6]. The point-source rejection already exceeds the level that will be provided by next generation adaptive optics systems working through the atmosphere. 
Research, Science and Technology of Brown Dwarfs and Exoplanets

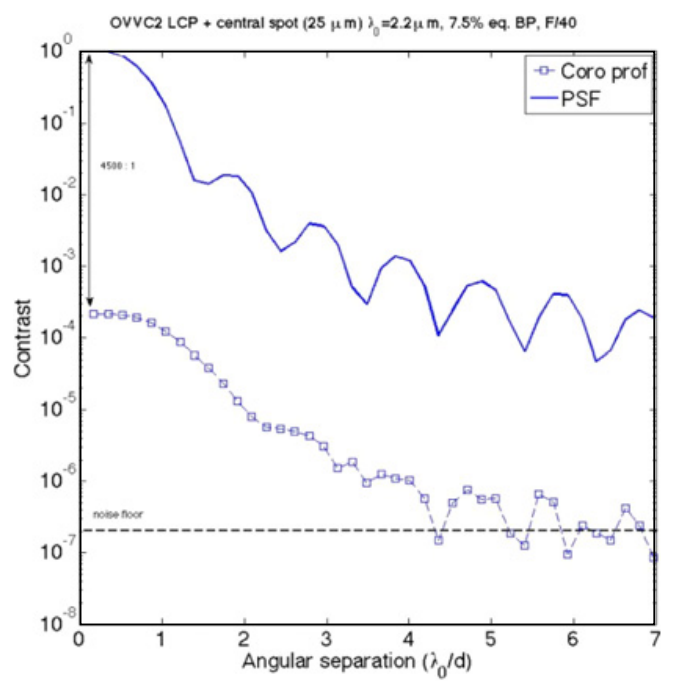

Figure 3. Laboratory contrast of one of our infrared vortex devices.

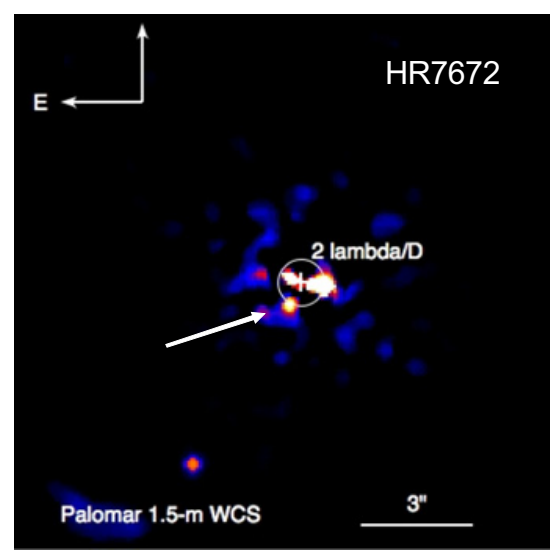

Figure 4. Image of a brown dwarf companion (HR7672b) at $\sim 2.5 \lambda / \mathrm{D}$ obtained with a four quadrant phase mask on the well-corrected subaperture [3].

\section{ON-SKY RESULTS}

After manufacture and testing, we installed our coronagraphic phase masks and a clear Lyot stop in the PHARO camera at Palomar, and carried out on-sky test observations using our well-corrected subaperture [8]. The well-corrected subaperture, a clear $1.5 \mathrm{~m}$ off-axis subaperture matched to the adaptive optics system's deformable mirror, typically provides Strehl ratios of $\sim 90 \%$ in the Ks band. This system has quickly proven itself capable of detecting faint companions [3] and disks [9] very close to stars (Figures 4 and 5), because of the combination of small intrinsic inner working angles provided by the phase masks, and the high wavefront quality provided by the well-corrected subaperture. These capabilities have even recently allowed the detection of the three planets around HR8799 with the well corrected subaperture and a vector vortex coronagraph [10]. 


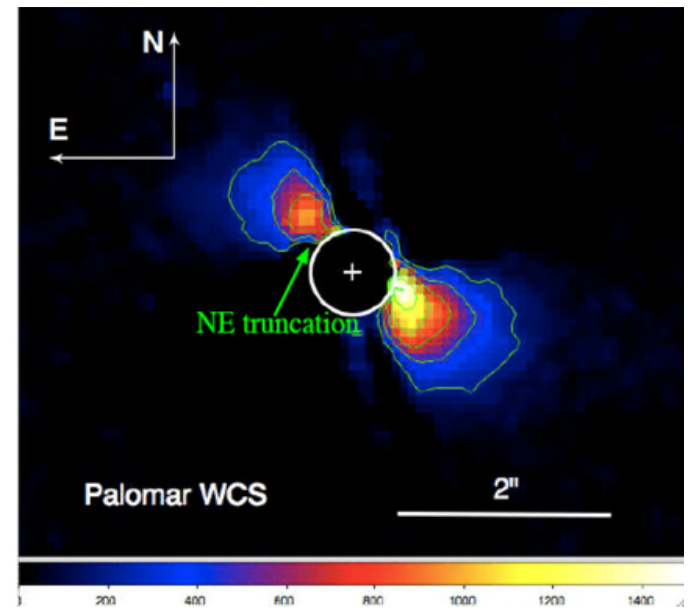

Figure 5. Image of the HD32297 debris disk obtained with a four-quadrant phase mask on the well-corrected subaperture [9].

\section{SUMMARY}

Because of small inner working angles and high throughput, phase masks hold great promise for small-angle faint companion detection. Moreover, based on their demonstrated performance with the Palomar well-corrected subaperture, they have started to live up to their potential. In particular, the performance already achieved with our small aperture at Palomar is competitive with that of much larger telescopes corrected with current generation adaptive optics systems. As such, once implemented on large telescopes, the next-generation of extreme adaptive optics systems should benefit markedly from the use of phase masks. Space-based coronagraphy could also benefit greatly from the reduced inner working angles provided by phase masks, allowing smaller telescopes to be used in coronagraphic missions.

This work was carried out at the Jet Propulsion Laboratory, California Institute of Technology, under contract with the National Aeronautics and Space Administration, and is based on observations obtained on the Hale Telescope, Palomar Observatory, as part of a continuing collaboration between the California Institute of Technology, NASA/JPL and Cornell University.

(c) 2009 California Institute of Technology. Government sponsorship acknowledged.

\section{References}

[1] P. Haguenauer et al., Proc. SPIE 5905, 59050S (2005)

[2] P. Haguenauer et al., Proc. SPIE 6265, 62651G, (2006)

[3] E. Serabyn et al., Astrophys. J. 696, 40 (2009)

[4] D. Mawet et al., Optics Express 17, 1902 (2009)

[5] D. Mawet et al., Proc. SPIE 7440, 74400X (2009)

[6] D. Mawet et al., Astrophys. J., in press (2010)

[7] D. Rouan, P. Riaud, A. Boccaletti, Y. Clenet \& A. Labeyrie, P.A.S.P. 112, 1479 (2000)

[8] E. Serabyn et al., Astrophys. J. 658, 1386 (2007)

[9] D. Mawet, E. Serabyn, K. Stapelfeldt \& J. Crepp, Astrophys. J. 702, L47 (2009)

[10] E. Serabyn et al., submitted (2010) 